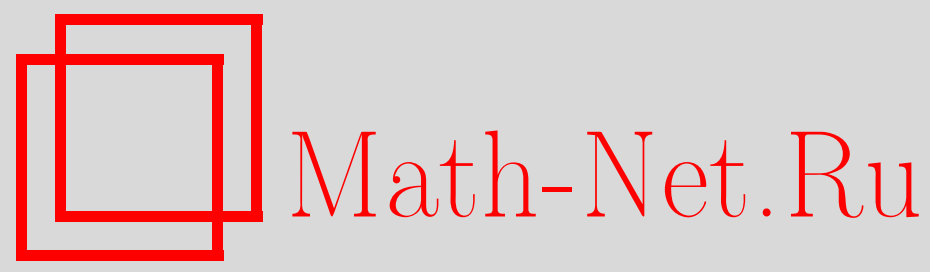

С. Ф. Каморников, Л. П. Авдашкова, Радикальные дистрибутивные функторы, Матем. заметки, 2000, том 68, выпуск $1,91-97$

DOI: https://doi.org/10.4213/mzm922

Использование Общероссийского математического портала Math-Net.Ru подразумевает, что вы прочитали и согласны с пользовательским соглашением http://www.mathnet.ru/rus/agreement

Параметры загрузки:

IP : 52.23 .180 .231

26 апреля 2023 г., $16: 26: 50$

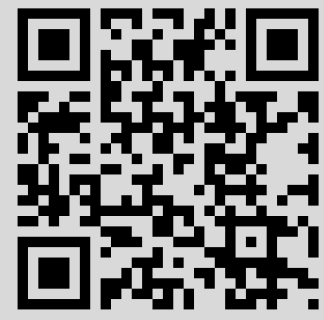


УДК 512.542

\title{
РАДИКАЛЬНЫЕ ДИСТРИБУТИВНЫЕ ФУНКТОРЫ
}

\author{
С. Ф. Каморников, Л. П. Авдашкова
}

Исследуются свойства дистрибутивных функторов на решетке субнормальных подгрупп. Выделяется класс радикальных дистрибутивных функторов, устанавливается связь таких функторов с классами Фиттинга, описьваются все классы Фиттинга, индуцирующие радикальный дистрибутивный функтор.

Библиограффия: 7 названий.

1. Введение. При изучении перестановочных свойств субнормальных подгрупп [1] и исследовании подгруппового и нормального строения конечных групп с системой косубнормальных подгрупп [2] существенным образом используются технические возможности дистрибутивных функторов, т.е. таких подгрупповых функторов, которые согласованы с изоморфизмами групп и индуцируют верхний эндоморфизм на решетке субнормальных подгрупп каждой группы.

В последнее время значительньй прогресс достигнут в части исследования дистрибутивных функторов, перестановочных с гомоморфизмами групп (см., например, работы [3], [4], где такие функторы называются операторами Виландта). В частности, в [4] установлена тесная связь этих функторов с формациями Фиттинга и показанно, что действие их на каждой групе заключается в выделении ее $\mathfrak{F}$-корадикала для некоторой формации Фиттинга $\mathfrak{F}$. Таким образом, в [4] описание операторов Виландта сведено к описанию формаций Фиттинга, обладающих тем свойством, что $\langle H, K\rangle^{\mathfrak{F}}=\left\langle H^{\mathfrak{F}}, K^{\mathfrak{F}}\right\rangle$ для всех $H, K \in \operatorname{sn}(G)$.

Однако не все дистрибутивные функторы перестановочны с гомоморфизмами групा. Первьй пример таких функторов построен Виландтом в [2]. Пусть $\mathfrak{X}$ - класс всех простых неабелевых групп, $\mathfrak{H}$ - класс всех тех групп, которые представимы в виде конечных прямых произведений групп из класса $\mathfrak{X}$. Виландт [2] показал, что отображение $\Theta$, сопоставляющее каждой группе ее наибольшую нормальную $\mathfrak{H}$-подгруппу, задает дистрибутивный функтор.

Этот пример приводит нас к идее радикальных дистрибутивных функторов, которые и изучаются в настояшей работе.

2. Постановка задачи. В работе рассматриваются только конечные грушшы, используются определения и обозначения, принятые в книгах [5], [6]. Следуя Виландту [2], дистрибутивным функтором будем назьвать отображение $\Theta$, сопоставляющее каждой группе $G$ ее некоторую подгруппу $\Theta(G)$ и обладающее следующими свойствами:

1) $f(\Theta(G))=\Theta(f(G))$ для любого изоморфизма $f$ группы $G$;

2) $\Theta(\langle X, Y\rangle)=\langle\Theta(X), \Theta(Y)\rangle$ для любых $X, Y \in \operatorname{sn}(G)$. 
Дистрибутивньй функтор $\Theta$ назьвается радикальным, если выполняется условие

3) $\Theta(X)=X \cap \Theta(G)$ для любой подгруппы $X \in \operatorname{sn}(G)$.

Существует прямая зависимость между радикальными дистрибутивньми функторами и классами Фиттинга. Употребление слова "радикальньй" здесь не случайно. Оно отражает связь радикальных функторов с радикальными классами. Напомним, что класс групп $\mathfrak{X}$ назьвается радикальным или классом Фиттинга, если он является нормально наследственным, т.е. из $G \in \mathfrak{X}$ и из того, что $G=A B$, где $A$ и $B$ - нормальные $\mathfrak{X}$-подгрупшы из $G$, всегда следует $G \in \mathfrak{X}$. Если $\mathfrak{X}$-класс Фиттинга, то произведение всех нормальных $\mathfrak{X}$-подгрупп группы $G$ назьвается ее $\mathfrak{X}$-радикалом и обозначается через $G_{\mathfrak{X}}$.

ТЕОрема 2.1. Пусть $\Theta-$ радикальный дистрибутивный функтор. Тогда

1) множество $\mathfrak{F}=\{H \mid \Theta(H)=H\}$ является классом Фиттинга;

2) для любой әруппы $G$ подгруппа $\Theta(G)$ совпадает с ее $\mathfrak{F}$-радикалом $G_{\mathfrak{F}}$.

ДокАЗАтЕльство. Так как функтор $\Theta$ обладает свойством 1 ), множество $\mathfrak{F}$ является классом, т.е. вместе с каждой своей групой $G$ содержит все группы, изоморфные $G$.

Пусть $G \in \mathfrak{F}$, т.е. $\Theta(G)=G$. Если $N$ - нормальная подгруппа группы $G$, то, учитьвая свойство 3), имеем $\Theta(N)=N \cap \Theta(G)=N \cap G=N$. Следовательно, $N \in \mathfrak{F}$. Значит, $\mathfrak{F}$ - нормально наследственный класс.

Пусть теперь $N, K$ - нормальные $\mathfrak{F}$-подгруппы группы $G=N K$. Так как $\Theta(N)=N$, $\Theta(K)=K$, то

$$
\Theta(G)=\Theta(N K)=\Theta(\langle N, K\rangle)=\langle\Theta(N), \Theta(K)\rangle=\langle N, K\rangle=G
$$

Следовательно, $G \in \mathfrak{F}$. Итак, $\mathfrak{F}-$ класс Фиттинга.

Так как $G_{\mathfrak{F}} \in \mathfrak{F}$, из определения класса $\mathfrak{F}$ имеем $\Theta\left(G_{\mathfrak{F}}\right)=G_{\mathfrak{F}}$. С другой стороны, по свойству 3) оператора $\Theta$ имеем $\Theta\left(G_{\mathfrak{F}}\right)=G_{\mathfrak{F}} \cap \Theta(G)$. Отсюда $G_{\mathfrak{F}}=G_{\mathfrak{F}} \cap \Theta(G)$. Итак, $G_{\mathfrak{F}} \subseteq \Theta(G)$. Ввиду свойства 3) имеем $\Theta(\Theta(G))=\Theta(G) \cap \Theta(G)=\Theta(G)$, т.е. $\Theta(G) \in \mathfrak{F}$. Ввиду свойства 1$) \Theta(G)$ является нормальной подгруппой групшы $G$. Теперь из определения $\mathfrak{F}$-радикала получаем $\Theta(G) \subseteq G_{\mathfrak{F}}$. Значит, $G_{\mathfrak{F}}=\Theta(G)$. Теорема доказана.

Теорема 2.1 связывает с каждым радикальным дистрибутивньгм функтором $\Theta$ класс Фиттинга $\mathfrak{F}=\{H \mid \Theta(H)=H\}$ и показьвает, что действие $\Theta$ на группе заключается в выделении ее $\mathfrak{F}$-радикала.

Обратно, каждому классу Фиттинга $\mathfrak{F}$ соответствует функция $\operatorname{rad}_{\mathfrak{F}}: G \rightarrow G_{\mathfrak{F}}$, которая удовлетворяет условиям 1) и 3 ). Если она также удовлетворяет условию 2), то $\operatorname{rad}_{\mathfrak{F}}-$ радикальньй дистрибутивньй функтор.

Таким образом, каждьй радикальньй дистрибутивньй функтор $\Theta$ имеет вид $\Theta=$ $\operatorname{rad}_{\mathfrak{F}}$ для некоторого класса Фиттинга $\mathfrak{F}$ и задача описания радикальных дистрибутивных функторов сводится к описанию классов Фиттинга, обладающих тем свойством, что

$$
\langle H, K\rangle_{\mathfrak{F}}=\left\langle H_{\mathfrak{F}}, K_{\mathfrak{F}}\right\rangle
$$

для всех $H, K \in \operatorname{sn}(G)$. Ниже в теореме 4.1 мы описываем все классы Фиттинга, которые обладают указанным свойством. 
3. Вспомогательные леммы. Доказательство следующей леммы можно найти в [6].

Лемма 3.1. Пусть $\mathfrak{F}$ - класс Фиттинга, $G$ - әруппа. Тогда

а) если $N \in \operatorname{sn}(G)$, mo $N_{\mathfrak{F}}=N \cap G_{\mathfrak{F}}$;

в) если $N_{1}, N_{2}, \ldots, N_{t}-$ нормальные подгруппь группь $G=N_{1} N_{2} \cdots N_{t}$, то

$$
G_{\mathfrak{F}} /\left(\prod_{i=1}^{t}\left(N_{i}\right)_{\mathfrak{F}}\right) \subseteq Z\left(G / \prod_{i=1}^{t}\left(N_{i}\right)_{\mathfrak{F}}\right)
$$

Лемма 3.2. Пусть $\mathfrak{F}$ - класс Фиттинга такой, что каждая группа из $\mathfrak{F}$ не имеет абелевых композииионных факторов. Если $G=H K$, где $H$ и $K$ - нормальные подгруппьи из $G$, то $G_{\mathfrak{F}}=H_{\mathfrak{F}} K_{\mathfrak{F}}$.

ДокАЗАТЕЛЬСТво. Ввиду леммы $3.1 H_{\mathfrak{F}} K_{\mathfrak{F}} \subseteq G_{\mathfrak{F}}$ и $G_{\mathfrak{F}} / H_{\mathfrak{F}} K_{\mathfrak{F}} \subseteq Z\left(G / H_{\mathfrak{F}} K_{\mathfrak{F}}\right)$. Так как $G_{\mathfrak{F}} \in \mathfrak{F}$ и все композиционные факторы $G_{\mathfrak{F}}$ неабелевы, то $G_{\mathfrak{F}} / H_{\mathfrak{F}} K_{\mathfrak{F}}=1$, т.е. $G_{\mathfrak{F}}=H_{\mathfrak{F}} K_{\mathfrak{F}}$. Лемма доказана.

Лемма 3.3. Пусть $\mathfrak{F}$ - класс Фиттинга такой, что каждая группа из $\mathfrak{F}$ не имеет абелевых композичионных факторов. Пусть $H$ и $K$ - субнормальные подгруппы группь $G u K \subseteq N_{G}(H)$. Тогда $(H K)_{\mathfrak{F}}=H_{\mathfrak{F}} K_{\mathfrak{F}}$.

ДоКАЗАТЕЛЬСТВо. Не нарушая общности рассуждений, можем считать $G=H K$. Применим индукцию по длине $j(H K-K)$ композиционной $(H K-K)$-цепи. Если $j(H K-K)=0$, то $H K=K$, т.е. $H \subseteq K$. Так как класс $\mathfrak{F}$ является нормально наследственньм, то $H_{\mathfrak{F}} \subseteq K_{\mathfrak{F}}$. Отсюда имеем $G_{\mathfrak{F}}=K_{\mathfrak{F}}=H_{\mathfrak{F}} K_{\mathfrak{F}}$.

Предположим, что $t=j(H K-K)>0$. Будем считать, что лемма верна для всех тех субнормальных подгрупा $X$ и $Y$ группы $G$, которые удовлетворяют условиям $Y \subseteq N_{G}(X)$ и $j(X Y-Y)<t$. Пусть $H K=K_{0} \supseteq K_{1} \supseteq \cdots \supseteq K_{t}=K$ - композиционная $(H K-K)$-цепь. Рассмотрим группу $K_{1}$. Так как $K \subseteq K_{1} \subseteq H K$, ввиду тождества Дедекинда $K\left(K_{1} \cap H\right)=K_{1} \cap K H=K_{1}$. Кроме того, $K \subseteq N_{K}\left(K_{1} \cap H\right)$ и $j\left(K_{1}-K\right)=t-1<t$. Следовательно, ввиду предположения индукции

$$
\left(K_{1}\right)_{\mathfrak{F}}=K_{\mathfrak{F}}\left(K_{1} \cap H\right)_{\mathfrak{F}}
$$

Так как $\left(K_{1} \cap H\right)_{\mathfrak{F}} \subseteq H_{\mathfrak{F}}$, то

$$
H_{\mathfrak{F}} K_{\mathfrak{F}}=H_{\mathfrak{F}}\left(K_{1} \cap H\right)_{\mathfrak{F}} K_{\mathfrak{F}}=H_{\mathfrak{F}}\left(K_{1}\right)_{\mathfrak{F}} .
$$

Ввиду леммы 3.2 имеем $G_{\mathfrak{F}}=\left(H K_{1}\right)_{\mathfrak{F}}=H_{\mathfrak{F}}\left(K_{1}\right)_{\mathfrak{F}}$. Отсюда следует, что $H_{\mathfrak{F}} K_{\mathfrak{F}}=G_{\mathfrak{F}}$. Лемма доказана.

Лемма 3.4. Пусть $\mathfrak{F}$ - класс Фиттинга такой, что каждая группа из $\mathfrak{F}$ не имеет абелевых композииионных факторов. Пусть $H$ и $K$ - субнормальные подгруппь зруппь $G$. Eсли $H K=K H, m o(H K)_{\mathfrak{F}}=H_{\mathfrak{F}} K_{\mathfrak{F}}$. 
ДокАЗАТЕЛЬСтво. Очевидно, подгрупша $K$ субнормальна в $H K$. Применим индукцию по длине $j(H K-K)$ композиционной $(H K-K)$-цепи. Ввиду леммы $3.3(H K)_{\mathfrak{F}}=$ $H_{\mathfrak{F}} K_{\mathfrak{F}}$, если $j(H K-K)<1$.

Итак, считаем в дальнейшем, что лемма верна для субнормальных подгрупा $X$ и $Y$ группы $G$ таких, что $j(X Y-Y)<m$. Пусть

$$
H K=K_{0} \supseteq K_{1} \supseteq \cdots \supseteq K_{m}=K
$$

- композиционная $(H K-K)$-цепь. Рассмотрим групшу $K_{1}$. Из $H K=K H$ и $K \subseteq K_{1}$ следует ввиду тождества Дедекинда, что $K\left(K_{1} \cap H\right)=K_{1} \cap K H=K_{1}$. Так как $j\left(K_{1}-K\right)=m-1<m$, по индукции $\left(K_{1}\right)_{\mathfrak{F}}=K_{\mathfrak{F}}\left(K_{1} \cap H\right)_{\mathfrak{F}}$. Из нормальной наследственности класса $\mathfrak{F}$ следует, что $\left(K_{1} \cap H\right)_{\mathfrak{F}} \subseteq H_{\mathfrak{F}}$. Следовательно,

$$
\left(K_{1}\right)_{\mathfrak{F}} H_{\mathfrak{F}}=K_{\mathfrak{F}}\left(K_{1} \cap H\right)_{\mathfrak{F}} H_{\mathfrak{F}}=K_{\mathfrak{F}} H_{\mathfrak{F}} .
$$

Очевидно, $K H=K_{1} H$. Ввиду леммы $3.3\left(K_{1} H\right)_{\mathfrak{F}}=\left(K_{1}\right)_{\mathfrak{F}} H_{\mathfrak{F}}$. Значит,

$$
(H K)_{\mathfrak{F}}=\left(H K_{1}\right)_{\mathfrak{F}}=\left(K_{1}\right)_{\mathfrak{F}} H_{\mathfrak{F}}=K_{\mathfrak{F}} H_{\mathfrak{F}} .
$$

Лемма доказана.

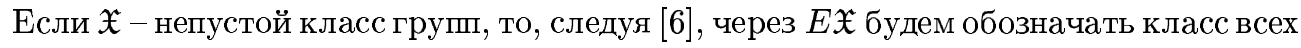
групп, обладающих субнормальными рядами, все факторы которых принадлежат $\mathfrak{X}$. В частности, если $\mathfrak{X}$ - непустой класс простых групп, то $E \mathfrak{X}$ - это класс всех тех групп, все композиционные факторы которых входят в $\mathfrak{X}$. Простая проверка показьвает, что в этом случае $E \mathfrak{X}-$ формация Фиттинга.

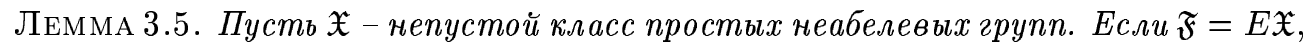
mо $\operatorname{rad}_{\mathfrak{F}}-$ радикальный дистрибутивный функтор.

ДокАЗАтЕльство. Допустим, что лемма неверна. Тогда найдется хотя бы одна группа, обладающая субнормальными подгруппами, для которых не вьполняется равенство (*). Пусть $G$ - группа наименьшего порядка с таким свойством. Тогда в $G$ найдутся субнормальные подгрупшы $H$ и $K$ такие, что $G=\langle H, K\rangle$ и $G_{\mathfrak{F}} \neq\left\langle H_{\mathfrak{F}}, K_{\mathfrak{F}}\right\rangle$. Пусть $N$ - минимальная нормальная подгруппа группы $G$. Рассмотрим два случая.

1. Пусть $N \in \mathfrak{F}$. Тогда ввиду выбора групшы $G$ имеем

$$
(G / N)_{\mathfrak{F}}=\left\langle(H N / N)_{\mathfrak{F}},(K N / N)_{\mathfrak{F}}\right\rangle .
$$

Так как класс $\mathfrak{F}$ замкнут относительно расширений, то

$$
(G / N)_{\mathfrak{F}}=G_{\mathfrak{F}} / N, \quad(H N / N)_{\mathfrak{F}}=H_{\mathfrak{F}} N / N, \quad(K N / N)_{\mathfrak{F}}=K_{\mathfrak{F}} N / N .
$$

Отсюда следует, что $G_{\mathfrak{F}}=\left\langle H_{\mathfrak{F}}, K_{\mathfrak{F}}\right\rangle N$. Так как $G_{\mathfrak{F}} \in \mathfrak{F}$, то

$$
G_{\mathfrak{F}}=H_{\mathfrak{F}} K_{\mathfrak{F}} N=\left(H \cap G_{\mathfrak{F}}\right)\left(K \cap G_{\mathfrak{F}}\right) N .
$$

Так как подгрупша $N$ характеристически проста, то $N=N_{1} \times \cdots \times N_{k}$, где $N_{i}$ - субнормальные неабелевы простые подгрупшы группы $G$. Ввиду леммы 1.8 из [2] подгруппа $N_{i}$ содержится либо в $H$, либо в $K$ для любого $i=1,2, \ldots, k$. Значит, $N \subseteq\left(H \cap G_{\mathfrak{F}}\right)\left(K \cap G_{\mathfrak{F}}\right)$. Следовательно, $G_{\mathfrak{F}}=H_{\mathfrak{F}} K_{\mathfrak{F}}$. Противоречие.

2. Пусть все минимальные нормальные подгруппы группы $G$ не принадлежат $\mathfrak{F}$. Тогда $G_{\mathfrak{F}}=1$. Так как класс $\mathfrak{F}$ является нормально наследственным, то $H_{\mathfrak{F}}=K_{\mathfrak{F}}=1$. Таким образом, $G_{\mathfrak{F}}=H_{\mathfrak{F}} K_{\mathfrak{F}}$. Снова пришли к противоречию. Лемма доказана. 
Лемма 3.6. Пусть $\mathfrak{F}-$ класс Фиттинга такой, что каждая группа из $\mathfrak{F}$ не имеет абелевых композичионных факторов. Тогда $\operatorname{rad}_{\mathfrak{F}}-$ радикальный дистрибутивный функтор.

ДокАЗАТЕльство. Пусть $G$ - групша наименьшего порядка, обладаюшая субнормальными подгруппами, для которых не вьполняется равенство $(*)$. Тогда в $G$ найдутся субнормальные подгрупшы $H$ и $K$ такие, что $G=\langle H, K\rangle, G_{\mathfrak{F}} \neq\left\langle H_{\mathfrak{F}}, K_{\mathfrak{F}}\right\rangle$. Пусть $\mathfrak{H}=E \mathfrak{F}$. По лемме 3.5 имеем $G_{\mathfrak{H}}=\left\langle H_{\mathfrak{H}}, K_{\mathfrak{H}}\right\rangle$. Очевидно,

$$
G_{\mathfrak{F}}=\left(G_{\mathfrak{H}}\right)_{\mathfrak{F}}, \quad H_{\mathfrak{F}}=\left(H_{\mathfrak{H}}\right)_{\mathfrak{F}}, \quad K_{\mathfrak{F}}=\left(K_{\mathfrak{H}}\right)_{\mathfrak{F}}
$$

Если $G_{\mathfrak{H}} \neq G$, то ввиду выбора групшы $G$ имеем

$$
G_{\mathfrak{F}}=\left(G_{\mathfrak{H}}\right)_{\mathfrak{F}}=\left\langle\left(H_{\mathfrak{H}}\right)_{\mathfrak{F}},\left(K_{\mathfrak{H}}\right)_{\mathfrak{F}}\right\rangle=\left\langle H_{\mathfrak{F}}, K_{\mathfrak{F}}\right\rangle
$$

Противоречие.

Значит, $G_{\mathfrak{H}}=G$, т.е. $G \in \mathfrak{H}$. В этом случае $H=H^{\prime}, K=K^{\prime}$. По теореме Виландта из [7] подгруппы $H$ и $K$ перестановочны. Значит, по лемме $3.4 G_{\mathfrak{F}}=\left\langle H_{\mathfrak{F}}, K_{\mathfrak{F}}\right\rangle$. Снова пришли к противоречию. Лемма доказана.

Лемма 3.7. Пусть $A$ и $H$ - группы такие, что $|A|=p$, а $H$ имеет нормальную подгруппу $R$ индекса $p$. Тогда группа $G=A \times H$ содержит нормальную подгрупnу $S$, обладающ,ую следующими свойствами:

1) $G=H S$

2) $H \cap S=R$;

3) $H \simeq S$;

4) $A \cap S=1$.

ДокАЗАТЕЛЬСТво. Из условия леммы следует, что $H / R=\langle x R\rangle$ для некоторого $p$-элемента $x \in G$. Тогда

$$
H=R \cup x R \cup \cdots \cup x^{p-1} R=\langle x\rangle R
$$

Пусть $A=\langle y\rangle$. Рассмотрим групу $S=\langle x y\rangle R$. Очевидно, $S \triangleleft G$.

Допустим, что $S=H$. Тогда из $x \in H$ и $x y \in H$ следует, что $y \in H$. Но это противоречит тому, что $A \cap H=1$. Если предположить, что $S=R$, то $x y \in R$, а значит, $y \in x^{p-1} R \subseteq H$. Снова приходим к противоречию. Если $S=G$, то $x \in S$. Тогда $S=\langle y\rangle \times R$, а значит, из свойств прямых произведений заключаем, что $x \in R$ и $H=R$. Полученное противоречие показьвает, что $S$ - максимальная подгруппа групы $G$, отличная от $H$. Поэтому $G=H S$.

Так как $R \subseteq H, R \subseteq S$ и подгруппа $R$ максимальна в $H$ и $S$, то из $H \neq S$ следует, что $H \cap S=R$.

Допустим, что $A \cap S \neq 1$. Тогда из $|A|=p$ имеем $A \subseteq S$. Из $x y \in S$ и $y \in S$ следует, что $x \in S$. Но тогда $S=H$ и мы приходим к противоречию.

Покажем теперь, что $H \simeq S$. Из (**) следует, что любой элемент группы $H$ представим в виде $x^{k} r$, где $0 \leqslant k<p, r \in R$. Рассмотрим отображение $f: H \rightarrow S$ такое, что $f\left(x^{k} r\right)=(x y)^{k} r$. Тогда, с одной стороны, на основании $(* *)$ имеем

$$
f\left(\left(x^{k} r_{1}\right)\left(x^{l} r_{2}\right)\right)=f\left(x^{k+l} r_{3}\right)=(x y)^{k+l} r_{3} .
$$


С другой стороны, так как $y \in Z(G)$, то

$$
f\left(\left(x^{k} r_{1}\right)\left(x^{l} r_{2}\right)\right)=(x y)^{k} r_{1}(x y)^{l} r_{2}=\left(x^{k} r_{1}\right)\left(x^{l} r_{2}\right) y^{k+l}=x^{k+l} r_{3} y^{k+l}=(x y)^{k+l} r_{3} .
$$

Следовательно, $f$ - гомоморфизм. Пусть $z \in \operatorname{Ker} f$. Тогда из $z \in H$ имеем, что $z=x^{k} r$ для некоторых $0 \leqslant k<p, r \in R$. С другой стороны, $f(z)=(x y)^{k} r=y^{k} x^{k} r=1$. Так как $G=A \times H$, то любой элемент $g$ из $G$ единственным образом представим в виде $g=a h$, где $a \in A, h \in H$. Следовательно, из $y^{k} x^{k} r=1$ имеем, в частности, что $x^{k} r=z=1$. Таким образом, $\operatorname{Ker} f=1$, а значит, $f$-изоморфизм. Лемма доказана.

Доказательство следующей леммы легко следует из леммы 3.7 (см. также [6, с. 565]).

ЛЕмма 3.8. Пусть $\mathfrak{F}-$ класс Фиттинга. Если группа $G \in \mathfrak{F}$ содержит композичионный фактор порядка $p$, то $\mathfrak{F}$ содержит чиклическую группу порядка р.

\section{4. Основной результат.}

ТЕОРема 4.1. Пусть $\mathfrak{F}-$ класс Фиттинга. Тогда и только тогда $\operatorname{rad}_{\mathfrak{F}}-p a \partial u-$ кальный дистрибутивный функтор, когда либо $\mathfrak{F}$ - класс всех групп, либо каждая группа из $\mathfrak{F}$ не имеет абелевых композичионных факторов.

ДокАЗАТЕЛЬСТво. Пусть $\mathfrak{F}$ - собственньй подкласс класса всех групп. Пусть $H-$ группа наименьшего порядка, не принадлежащая $\mathfrak{F}$. Так как $\mathfrak{F}$ - класс Фиттинга, то $H$ имеет единственную максимальную нормальную подгруппу $R$. Очевидно, $R=H_{\mathfrak{F}}$.

Предположим, что $\mathfrak{F}$ содержит некоторую группу, имеющую композиционньй фактор порядка $p$. Тогда ввиду леммы 3.8 группа $A$ порядка $p$ входит в $\mathfrak{F}$. Возможны три случая.

1. Пусть $H / R$ - абелева группа и $H / R \in \mathfrak{F}$. Тогда, не нарушая общности рассуждений, можем полагать, что $H / R \simeq A$.

Рассмотрим группу $G=A \times H$. Тогда ввиду леммы 3.7 в $G$ найдется нормальная подгруппа $S$ такая, что $G=S H, H \cap S=R, H \simeq S$ и $A \cap S=1$. Так как $H_{\mathfrak{F}}=R$, $H \simeq S$ и $R \subseteq S$, то $S_{\mathfrak{F}}=R$. Поэтому $H_{\mathfrak{F}} S_{\mathfrak{F}}=R$. С другой стороны, так как $A \in \mathfrak{F}$, то $G_{\mathfrak{F}} \supseteq R A$. Таким образом, $G_{\mathfrak{F}} \neq H_{\mathfrak{F}} S_{\mathfrak{F}}$ и мы приходим к противоречию с условием теоремы.

2. Пусть $H / R$ - абелева группа и $H / R$ не входит в $\mathfrak{F}$. Тогда $|H|=q$ для некоторого простого $q$, отличного от $p$. Пусть $H_{1}=H \mathrm{wr} A=[K] A$, где $K-$ база регулярного сплетения $H$ wr $A$. Если $\left(H_{1}\right)_{\mathfrak{F}} \neq 1$, то из $\left|H_{1}\right|=q^{p} p$ следует, что $\left(H_{1}\right)_{\mathfrak{F}}=A$. Но тогда $A$ - нормальная подгруппа в $H_{1}$, что невозможно. Значит, $\left(H_{1}\right)_{\mathfrak{F}}=1$.

Рассмотрим теперь группу $G_{1}=A \times H_{1}$. Ввиду леммы 3.7 в $G_{1}$ найдется нормальная подгруппа $S_{1}$ такая, что $G_{1}=S_{1} H_{1}, H_{1} \cap S_{1}=K, S_{1} \simeq H_{1}$ и $A \cap S_{1}=1$. В этом случае $\left(S_{1}\right)_{\mathfrak{F}}=1$ и $\left(G_{1}\right)_{\mathfrak{F}} \supseteq A$. Поэтому $\left(G_{1}\right)_{\mathfrak{F}} \neq\left(H_{1}\right)_{\mathfrak{F}}\left(S_{1}\right)_{\mathfrak{F}}=1$. Снова пришли к противоречию.

3. Пусть $H / R$ - неабелева группа. Пусть $H_{2}=H \operatorname{wr} A=\left[K_{1}\right] A$, где $K_{1}-$ база регулярного сплетения $H$ wr $A$. Так как $K_{1}$ - прямое произведение $p$ копий $L_{i}$ группы $H$, ввиду леммы $3.1\left(K_{1}\right)_{\mathfrak{F}}=\left(L_{1}\right)_{\mathfrak{F}} \times \cdots \times\left(L_{p}\right)_{\mathfrak{F}}$, где $\left(L_{i}\right)_{\mathfrak{F}} \simeq R$. Допустим, что $\left(H_{2}\right)_{\mathfrak{F}} \neq\left(K_{1}\right)_{\mathfrak{F}}$. Тогда из $\left(H_{2}\right)_{\mathfrak{F}} \cap K_{1}=\left(K_{1}\right)_{\mathfrak{F}}$ следует, что $\left|\left(H_{2}\right)_{\mathfrak{F}} /\left(K_{1}\right)_{\mathfrak{F}}\right|=p$. Поэтому групша $H_{2} /\left(K_{1}\right)_{\mathfrak{F}}$ содержит нормальную подгруппу порядка $p$, что невозможно, так как $H_{2} /\left(K_{1}\right)_{\mathfrak{F}} \simeq(H / R)$ wr $A$. Значит, $\left(H_{2}\right)_{\mathfrak{F}}=\left(K_{1}\right)_{\mathfrak{F}}$.

Рассмотрим группу $G_{2}=A \times H_{2}$. Ввиду леммы 3.7 в $G_{2}$ найдется нормальная подгруппа $S_{2}$ такая, что $G_{2}=S_{2} H_{2}, S_{2} \cap H_{2}=K_{1}, H_{2} \simeq S_{2}$ и $A \cap S_{2}=1$. Поэтому 
из $\left(K_{1}\right)_{\mathfrak{F}} \subseteq S_{2}$ следует, что $\left(S_{2}\right)_{\mathfrak{F}}=\left(K_{1}\right)_{\mathfrak{F}}$. Таким образом, $\left(S_{2}\right)_{\mathfrak{F}}\left(H_{2}\right)_{\mathfrak{F}}=\left(K_{1}\right)_{\mathfrak{F}}$, $\mathrm{a}\left(G_{2}\right)_{\mathfrak{F}} \supseteq A \times\left(K_{1}\right)_{\mathfrak{F}}$. Полученное противоречие и завершает доказательство первой части теоремы, т.е. если $\operatorname{rad}_{\mathfrak{F}}-$ радикальный дистрибутивный функтор, то либо $\mathfrak{F}-$ класс всех групп, либо каждая группа из $\mathfrak{F}$ не имеет абелевых композиционных факторов. Обратное утверждение следует из леммы 3.6. Теорема доказана.

\section{СПИСОК ЦИТИРОВАННОЙ ЛИТЕРАТУРЫ}

[1] Wielandt H. Vertauschbare nachinvariante Untergruppen // Abh. Math. Sem. Univ. Hamburg. 1957. V. 21. №1-2. P. 55-62.

[2] Wielandt H. Über das Erzeugnis paarweise kosubnormaler Untergruppen // Arch. Math. 1980. V. 35. № 1-2. P. 1-7.

[3] Каморников С. Ф. О некоторых свойствах формации квазинильпотентных групп // Матем. заметки. 1993. Т. 53. №2. С. 71-77.

[4] Каморников С.Ф., Шеметков Л.А. О корадикалах субнормальных подгрупп // Алгебра и логика. 1995. Т. 34. № 5. С. 493-513.

[5] Шеметков Л. А. Формации конечных групп. М.: Наука, 1978.

[6] Doerk K., Hawkes T. Finite soluble groups. Berlin-New York: Walter de Gruyter, 1992.

[7] Wielandt H. Eine Verallgemeinerung der invarianten Untergruppen // Math. Z. 1939. V. 45. P. 209-244.

(С. Ф. Каморников) Гомельский государственньй университет им. $\Phi$. Скорины

Поступило

(Л.П. Авдашкова) Гомельский кооперативный институт

28.12 .1998

Исправленный вариант

03.02 .2000 